\title{
Genetic and Phenotypic Characteristics of Staphylococcus aureus Isolates from Cystic Fibrosis Patients in Austria
}

\author{
Lilian Masoud-Landgraf ${ }^{a}$ Sophia Johler ${ }^{c} \quad$ Alexandra Badura ${ }^{a}$ Gebhard Feierla \\ Josefa Luxner ${ }^{\mathrm{a}} \quad$ Ute Wagner-Eibel $^{\mathrm{a}}$ Ernst Eber $^{\mathrm{b}} \quad$ Gernot Zarfel $^{\mathrm{a}}$ \\ Andrea J. Grisold ${ }^{a}$ \\ ${ }^{\mathrm{a}}$ Institute of Hygiene, Microbiology and Environmental Medicine, and ${ }^{\mathrm{b}}$ Respiratory and Allergic Disease Division, \\ Department of Paediatrics and Adolescent Medicine, Medical University of Graz, Graz, Austria; ' Institute for Food \\ Safety and Hygiene, Vetsuisse Faculty, University of Zurich, Zurich, Switzerland
}

\section{Key Words}

Staphylococcus aureus · Cystic fibrosis · spa typing ·

Antimicrobial resistance $\cdot$ Virulence genes

\begin{abstract}
Background: Cystic fibrosis (CF) is the most common lifelimiting inherited disease in Caucasian populations. While pathological changes can be seen in various organs, morbidity and mortality are mainly related to the respiratory tract, with patients suffering from chronic bronchopulmonary infections with characteristic pathogens including Staphylococcus aureus. Objectives: To date, there is only very limited data on the genetic and phenotypic characteristics of S. aureus in CF patients. Therefore, in our study, we characterized $58 \mathrm{~S}$. aureus isolates collected from CF patients in Austria by spa typing, DNA microarray profiling, as well as antimicrobial susceptibility testing in order to determine common genomic and antimicrobial resistance features. The tested strain collection exhibited high genomic diversity. Results: The 58 isolates were assigned to 16 clonal complexes and 48 spa types and differed greatly regarding their virulence and resistance gene profiles. The predominant clonal complexes were MLST CC30 (22\%), CC15 (16\%), CC45 (14\%), and CC5
\end{abstract}

(12\%), complexes that are highly prevalent worldwide among $S$. aureus strains isolated from humans colonized or infected with S. aureus. DNA microarray profiles showed a wide variety of genes encoding antimicrobial resistance and virulence factors such as various leukocidins, haemolysins, enterotoxins, exfoliative toxins, toxic shock syndrome toxin, as well as genes involved in adhesion and immune evasion. Conclusions: While a large number of strains exhibited resistance to one or several antimicrobial agents, methicillin-resistant S. aureus was found at a low prevalence of $3 \%(n=2)$ only. The two methicillin-resistant $S$. aureus isolates were assigned to CC152/t355 (SCCmecV) and CC5/t001 (SCCmecl). This is the first study to genetically characterize $S$. aureus isolates in CF patients in Austria.

(c) 2015 S. Karger AG, Basel

\section{Introduction}

Cystic fibrosis (CF) represents the most prevalent lifelimiting inherited disease in Caucasian populations [1]. While all organs expressing the cystic fibrosis transmembrane conductance regulator (including secretory cells, airways, liver, pancreas, and the reproductive tract) can

\section{KARGER 125}

(C) 2015 S. Karger AG, Basel

0025-7931/15/0895-0390\$39.50/0 
be affected, the most severe pathological changes are usually seen in the respiratory tract [2]. Airway pathology is characterized by obstruction, inflammation, and severe recurrent and/or chronic bacterial infections. One of the most prevalent organisms that can be detected in CF patients is Staphylococcus aureus, which was reported to represent one of the first pathogens infecting the airways of CF patients and persisting for months or even years [3]. During the last decades, infections with both methicillinsusceptible (MSSA) and methicillin-resistant S. aureus (MRSA) have increased considerably in CF patients in the United States. Prevalence of $S$. aureus in CF patients' respiratory cultures increased from 29\% in 1992 to $69 \%$ in 2012 , with the prevalence of MRSA increasing from $9 \%$ in 2002 to $25 \%$ in 2012 [4]. The same trend can be observed in European countries, whereas CF centres here report a lower rate of MSSA and MRSA in CF patients [5, 6]. Beside asymptomatic colonization of a patient, MSSA and MRSA can provoke a broad spectrum of infections, such as local infections up to endocarditis, pneumonia or sepsis. There are several publications reporting that MRSA-positive CF patients exhibit more complications, such as an increased hospitalization rate, an accelerated decline in lung function and an increased mortality rate $[7,8]$. Effective treatment of both MSSA and MRSA infections represents a major challenge, as $S$. aureus exhibits a huge repertoire of different resistance and virulence factors [9].

To date, it is still poorly understood which virulence factors are crucial in the pathogenesis of $S$. aureus infections in CF patients, and there is only little information on genomic and antimicrobial features of these strains. Microbiologic laboratories offer a number of different molecular typing methods, such as spa typing or more recently microarray techniques, which allow a more detailed view on the epidemiological as well as the genetic background of the investigated strains. Therefore, in this study, 58 isolates collected from $51 \mathrm{CF}$ patients in Austria were spa typed, and resistance and virulence gene profiles were investigated by DNA microarray analysis in order to gain new insights into the genomic and antimicrobial characteristics of $S$. aureus strains in CF patients.

\section{Materials and Methods}

\section{Bacterial Strains}

All S. aureus isolates included in this study were investigated at the CF Laboratory at the Institute of Hygiene, Microbiology and Environmental Medicine, Medical University of Graz, Austria. Samples originated from 51 CF patients and were collected over a period of 1 year in the CF centre at the Respiratory and Allergic Disease Division, Department of Paediatrics and Adolescent Medicine, Medical University of Graz. A total of 23 (45\%) patients were male and 28 (55\%) female. The patients' age ranged from 1 to 41 years, with a median age of 16 years. Samples included sputa $(n=46)$, throat swabs $(\mathrm{n}=10)$, and bronchoalveolar lavage fluids $(\mathrm{n}=2)$.

Species Identification and Antimicrobial Susceptibility Testing

$S$. aureus isolates were identified using a VITEK 2 (bioMérieux, Marcy l'Etoile, France) or MALDI-TOF MS instrument (Axima $^{\mathrm{TM}}$ Assurance, Shimadzu, Japan). Susceptibility testing was performed using both disk diffusion (Becton Dickinson, Heidelberg, Germany), according to the guidelines of the European Committee of Antimicrobial Susceptibility Testing, evaluating the susceptibility to penicillin $(1 \mu \mathrm{g})$, cefoxitin $(30 \mu \mathrm{g})$, erythromycin $(15 \mu \mathrm{g})$, clindamycin $(2 \mu \mathrm{g})$, gentamicin $(10 \mu \mathrm{g})$, and trimethoprim/sulfamethoxazole $(1.25 / 23.75 \mu \mathrm{g})$, and the VITEK 2 instrument (using the P580 card). Results were interpreted according to the guidelines of the European Committee of Antimicrobial Susceptibility Testing [10]. S. aureus with identical resistance phenotypes that had been isolated from the same patient were excluded from the study.

\section{Molecular Characterization by spa Typing and DNA}

Microarray

spa typing is a widely used technique for subtyping $S$. aureus for epidemiological surveillance in hospital and community settings. The spa typing method is based on sequencing of the polymorphic X region of the protein A gene (spa), present in almost all strains of $S$. aureus. We extracted DNA and performed spa typing as described by Ruppitsch et al. [11]. StaphyType DNA microarray (Clondiag Chip Technologies, Jena, Germany) was used according to the manufacturer's instructions to assign isolates to clonal complexes and to generate virulence and resistance gene profiles. This microarray system detects the presence/absence of genes conferring resistance to antimicrobial agents, as well as genes encoding for various virulence factors such as haemolysins, leukocidins, enterotoxins, and exfoliative toxins. In order to visualize the diversity of DNA microarray patterns, as well as strain relatedness, hybridization patterns were analysed using the SplitsTree software (www.splitstree.org), designed to compute unrooted phylogenetic networks from molecular sequence data [12]. DNA microarray gene profiles were converted to 'sequence-like' strings of information as previously described [13].

\section{Ethics Statement}

No ethical clearance was necessary, as the strains were isolated for diagnostic purposes and the patients were informed and consented in writing to the anonymized use of the isolates for study purposes.

\section{Results}

As 6 samples harboured $2(\mathrm{n}=5)$ or $3(\mathrm{n}=1)$ S. aureus isolates with different resistance phenotypes, a total of 58 S. aureus isolates collected from $51 \mathrm{CF}$ patients were included in the study. Among the investigated isolates, we frequently detected isolates resistant to one or more anti- 
Table 1. Antimicrobial resistance phenotypes of 58 S. aureus isolates collected from CF patients in Austria

\begin{tabular}{cllllll}
\hline \multirow{2}{*}{ (\%) } & \multicolumn{7}{l}{ Antimicrobial agents } & & \\
\cline { 2 - 7 } & OX & P & ERY & CLI & G & TM \\
\hline $22(38)$ & - & $\mathrm{X}$ & - & - & - & - \\
$14(24)$ & - & $\mathrm{X}$ & $\mathrm{X}$ & $(\mathrm{X})^{\mathrm{a}}$ & - & - \\
$8(14)$ & - & - & - & - & - & - \\
$3(5)$ & - & $\mathrm{X}$ & - & - & $\mathrm{X}$ & $\mathrm{X}$ \\
$2(3)$ & - & $\mathrm{X}$ & $\mathrm{X}$ & - & - & - \\
$1(2)$ & - & - & $\mathrm{X}$ & $(\mathrm{X})^{\mathrm{a}}$ & - & - \\
$1(2)$ & - & $\mathrm{X}$ & $\mathrm{X}$ & - & - & - \\
$1(2)$ & - & $\mathrm{X}$ & - & - & - & $\mathrm{X}$ \\
$1(2)$ & - & $\mathrm{X}$ & $\mathrm{X}$ & - & $\mathrm{X}$ & - \\
$1(2)$ & - & $\mathrm{X}$ & $\mathrm{X}$ & $(\mathrm{X})^{\mathrm{a}}$ & - & $\mathrm{X}$ \\
$1(2)$ & - & $\mathrm{X}$ & $\mathrm{X}$ & $(\mathrm{X})^{\mathrm{a}}$ & $\mathrm{X}$ & $\mathrm{X}$ \\
$1(2)$ & $\mathrm{X}$ & $\mathrm{X}$ & $\mathrm{X}$ & $\mathrm{X}$ & - & - \\
$1(2)$ & - & $\mathrm{X}$ & $\mathrm{X}$ & $\mathrm{X}$ & $\mathrm{X}$ & $\mathrm{X}$ \\
$1(2)$ & $\mathrm{X}$ & $\mathrm{X}$ & $\mathrm{X}$ & $(\mathrm{X})^{\mathrm{a}}$ & - & $\mathrm{X}$ \\
\hline Total, n & 2 & 49 & 21 & $2(18)^{\mathrm{a}}$ & 6 & 8 \\
\hline Total, $\%$ & 3 & 84 & 41 & $3(31)^{\mathrm{a}}$ & $(10)$ & 14 \\
\hline
\end{tabular}

$\mathrm{OX}=$ Oxacillin; $\mathrm{P}=$ penicillin; $\mathrm{ERY}=$ erythromycin; $\mathrm{CLI}=$ clindamycin; $\mathrm{G}=$ gentamicin; $\mathrm{TM}$ = trimethoprim .

a Inducible clindamycin resistance



Fig. 1. Genotypes and assignment to clonal complexes of $58 \mathrm{~S}$. aureus isolates from CF patients from Austria. Distribution: CC30 (13 isolates), CC15 (9 isolates), CC45 (8 isolates), CC5 (7 isolates), CC22 (4 isolates), CC398 (3 isolates), and CC8, CC9, and CC121 (2 isolates each). The unlabeled minor isolates were CC1, CC6, CC7, CC25, CC25/28, CC101, and CC152 with 1 isolate each, and there was 1 not assignable isolate.

suppl. material), $86 \%$ of the $S$. aureus isolates exhibited blaZ $/ R / I$, involved in resistance to penicillin, and 3\% harboured the mecA gene conferring resistance to methicillin. Genes involved in resistance to macrolides, lincosamides, and streptogramin were found in $11(19 \%$; erm $A)$, $1(2 \% ; \mathrm{ermB})$, and $6(10 \%$; erm $C)$ of the isolates. Concerning resistance to aminoglycosides, 11 isolates (19\%) exhibited a positive result for aacA-aphD (involved in resistance to gentamicin and tobramycin), 3 isolates (5\%) for addD (conferring resistance to tobramycin and neomycin), and 1 isolate (2\%) for aphA (involved in resistance to kanamycin and neomycin). The resistance gene $d f r A$ (conferring resistance to trimethoprim) was found in 5 isolates (9\%). In none of the S. aureus isolates, resistance genes for other agents, including genes conferring resistance to vancomycin, teicoplanin, linezolid, or mupirocin, were found. Concerning the detected virulence genes, a wide variety of genes encoding superantigens, including toxic shock syndrome toxin, major enterotoxins, and exfoliate toxins, were detected, as well as various haemolysins, leukocidins, and adhesins (online suppl. table 2). 
Fig. 2. SplitsTree depicting similarity of resistance and virulence gene profiles determined by DNA microarray for $58 \mathrm{~S}$. aureus isolates from CF patients in Austria. Clonal complexes are indicated for clusters comprised of 3 or more isolates. The 2 MRSA isolates are marked by black circles.

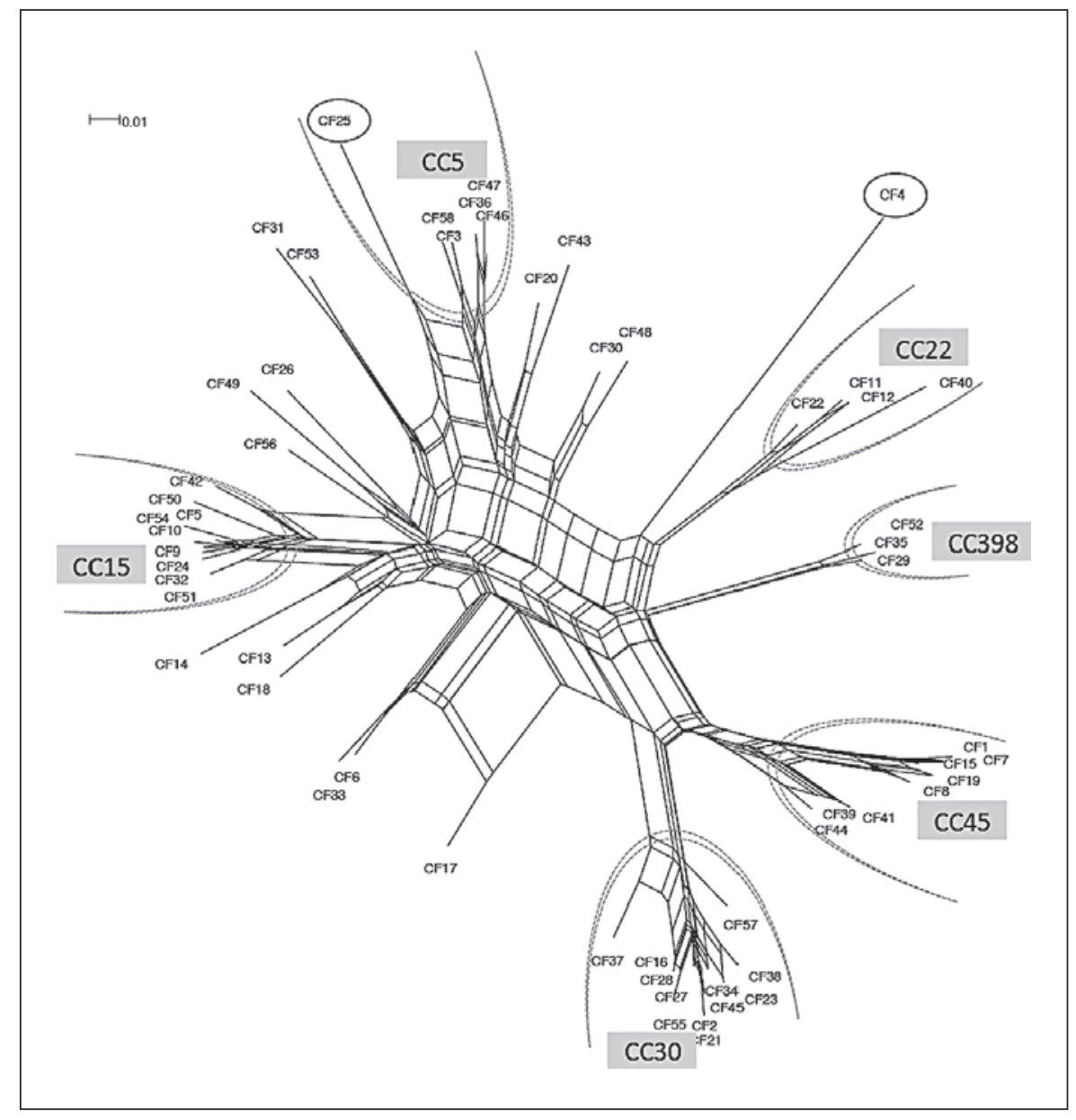

The gene coding for the pore-forming exotoxin PantonValentine leukocidin (PVL) could not be detected in any of the isolates. Assignment to agr types showed the following distribution: 27 isolates (47\%) belonged to agr I, $18(31 \%)$ to $a g r$ II, 15 (26\%) to $a g r$ III, and $10(17 \%)$ to $a g r$ IV. A comprehensive list of all microarray results, including genes encoding haemolysins, leukocidins, adhesins, as well as proteins involved in immune evasion, is provided in the supplementary material.

\section{Discussion}

Within the last years, several studies have reported a dramatic increase in MRSA strains isolated from CF patients, with MRSA rates of up to 25\% [4]. While for many decades, MRSA was solely associated with health careassociated transmission and infections, so-called community-acquired MRSA (CA-MRSA) can now be found both in the community but also in the hospital setting [ 5 , 6]. It has been controversially discussed if the increase in CA-MRSA, which frequently carry PVL, represents a new threat to CF patients $[14,15]$. PVL has been epidemiologically associated with severe skin and soft tissue infections, up to necrotizing pneumonia or severe sepsis [16].

In this study, MRSA could be detected in only 2 patients (3\%), and none of the investigated strains was positive for PVL. A similar result was also reported by Campana et al. [14] who investigated MRSA isolates from 9 Italian CF centres. The MRSA isolate carrying SCCmec V was assigned to CC152/t355, which was recently detected among CA-MRSA in Austria $[17,18]$. The MRSA isolate carrying SCCmecI was assigned to CC5/t001, which was reported in association with a hospital-acquired MRSA lineage (presumed Southern Germany clone) [19]. The overall low percentage of MRSA detected in our study is in accordance with previous reports describing constantly low local MRSA rates [17]. In addition, the CF centre 
at which the samples were collected follows a strict hygiene regimen to avoid person-to-person transmission. This includes single rooms for inpatients, as well as a strict separation of outpatients with known colonization with Pseudomonas spp., Burkholderia spp., MRSA, and nontuberculous mycobacteria. It is conceivable that the fact that each $S$. aureus strain was found only once among CF patients in our study is at least partly due to this strict hygiene regimen.

We did not only detect MRSA but also a multitude of strains resistant to penicillin, erythromycin, clindamycin, gentamicin, and trimethoprim. Only $14 \%$ of the isolates in this study were susceptible to all tested antimicrobial agents. The high rate of resistant strains mirrors a high selective pressure due to the antibiotic regimens that were used to treat CF patients. In the local CF centre, prophylactic flucloxacillin to prevent infection with $S$. aureus or chronic maintenance therapy with oral antistaphylococcal antibiotics are not used, in accordance with current guidelines [20]. However, as in many other CF centres and in line with agreed treatment standards, maintenance therapy with azithromycin was used in a number of patients chronically infected with Pseudomonas aeruginosa due to the dual effect of macrolides on infection and inflammation, despite concerns regarding the induction of resistance. In addition, it is standard to recommend the use of amoxicillin/clavulanic acid or other antistaphylococcal antibiotics (e.g. flucloxacillin) for a minimum of 2 weeks in case of viral respiratory tract infections, and for at least 4 weeks with pulmonary exacerbations if $S$. aureus is suspected to be the cause of the exacerbation. When a patient with pulmonary exacerbation has to be admitted for intravenous antibiotic therapy and S. aureus is isolated, an antistaphylococcal antibiotic is added to the treatment regimen.

The 58 S. aureus strains investigated in this study were assigned to a wide variety of clonal complexes and spa types and exhibited pronounced diversity regarding resistance and virulence gene profiles. All clonal complexes detected in this study can be commonly found among $S$. aureus strains isolated from humans colonized or infected with $S$. aureus $[13,21,22]$. Furthermore, there is evidence that the main reservoir of the $S$. aureus isolates is not the clinical setting, due to the predominance of MLST CC30 and CC15, described as community-associated S. aureus [23].

There are reports that after the first acquisition of $S$. aureus, these isolates persist for a longer period. In a study by Hirschhausen et al. [24], long-term persistence of $>5$ years with the same individual and isogenic $S$. aureus strain was detected. In vivo adaption processes could be observed, but the adaption processes seem to be very complex and might be influenced by individual host factors.

Some limitations of the study have to be addressed. While knowing the local antibiotic regimen, for this study, no detailed information on the antibiotic therapy of each individual CF patient or the clinical status at the day of sampling was retrieved. Beside that, this study was conducted in only a single CF centre in Austria and might be influenced by geographic or epidemiological factors. However, based on the fact that the same individual $S$. aureus strain might continuously colonize a CF patient, further studies could focus on the impact of these microbiological findings concerning the clinical status in these patients.

\section{Conclusions}

To conclude, this study provides for the first time information on the genomic background of $S$. aureus strains isolated from CF patients in Austria. In contrast to the high prevalence of MRSA reported among $S$. aureus isolates from CF patients in the US, only few MRSA isolates were detected (3\%). The S. aureus isolates investigated in this study exhibited high genetic diversity with regard to clonal complexes, spa types, as well as resistance and virulence gene profiles. This indicates that acquisition of these strains more likely occurred in the community setting than by patient-to-patient transmission.

References

\footnotetext{
1 O’Sullivan BP, Freedman SD: Cystic fibrosis. Lancet 2009;373:1891-1904.

2 Ratjen F, Doring G: Cystic fibrosis. Lancet 2003;361:681-689.

3 Saiman L: Microbiology of early CF lung disease. Paediatr Respir Rev 2004;5(suppl A):S367-S369.

4 Champion EA, Miller MB, Popowitch EB Hobbs MM, Saiman L, et al: Antimicrobial susceptibility and molecular typing of MRSA in cystic fibrosis. Pediatr Pulmonol 2014;49: 230-237.

5 Goss C, Muhlebach M: Review: Staphylococcus aureus and MRSA in cystic fibrosis. J Cyst Fibros 2011;10:298-306.

-6 Rosenthal VD, Bijie H, Maki DG, Mehta Y, Apisarnthanarak A, et al: International Nosocomial Infection Control Consortium (INICC) report, data summary of 36 countries, for 2004-2009. Am J Infect Control 2012;40: 396-407.
} 
7 Ren CL, Morgan WJ, Konstan MW, Schechter MS, Wagener JA, et al: Presence of methicillin resistant Staphylococcus aureus in respiratory cultures from cystic fibrosis patients is associated with lower lung function. Pediatr Pulmonol 2007;42:513-518.

8 Dasenbrook EC, Merlo CA, Diener-West M, Lechtzin N, Boyle MP: Persistent methicillinresistant Staphylococcus aureus and rate of $\mathrm{FEV}_{1}$ decline in cystic fibrosis. Am J Respir Crit Care Med 2008;178:814-821.

$\checkmark 9$ Muhlebach MS, Miller M, LaVange LM, Mayhew G, Goodrich JS, et al: Treatment intensity and characteristics of MRSA infection in CF. J Cyst Fibros 2011;10:201-206.

10 The European Committee on Antimicrobial Susceptibility Testing: Breakpoint tables for interpretation of MICs and zone diameters, version 2.0, 2012-01-01.

-11 Ruppitsch W, Indra A, Stoger A, Mayer B, Stadlbauer S, et al: Classifying spa types in complexes improves interpretation of typing results for methicillin-resistant Staphylococcus aureus. J Clin Microbiol 2006;44:24422448.

12 Huson DH, Bryant D: Application of phylogenetic networks in evolutionary studies. Mol Biol Evol 2006;23:254-267.
13 Wattinger L, Stephan R, Layer F, Johler S: Comparison of Staphylococcus aureus isolates associated with food intoxication with isolates from human nasal carriers and human infections. Eur J Clin Microbiol Infect Dis 2012;31: 455-464.

14 Campana S, Cocchi P, Doring G, Taccetti G, Moroney SM: Emergence of an epidemic clone of community-associated methicillinresistant Panton-Valentine leucocidin-negative Staphylococcus aureus in cystic fibrosis patient populations. J Clin Microbiol 2007;45: 3146, author reply 3146-3147.

15 Taccetti G, Cocchi P, Festini F, Braggion C, Campana S: Community-associated meticillin-resistant Staphylococcus aureus. Lancet 2010;376:767-768.

16 Gillet-Vittori L, Afanetti M, Dupont A, Gondon E, Dupont D: Life-threatening PantonValentine leukocidin-associated staphylococ cal infections in children. A broad spectrum of clinical presentations. Arch Pediatr 2014; 21:1220-1225.

17 Grisold AJ, Zarfel G, Stoeger A, Feierl G, Raggam RB, et al: Emergence of community-associated methicillin-resistant Staphylococcus aureus (CA-MRSA) in southeast Austria. J Infect 2009;58:168-170.

$>18$ Krziwanek K, Luger C, Sammer B, Stumvoll $\mathrm{S}$, Stammler M, et al: PVL-positive MRSA in Austria. Eur J Clin Microbiol Infect Dis 2007; 26:931-935.
19 Cookson BD, Robinson DA, Monk AB, Murchan S, Deplano A, et al: Evaluation of molecular typing methods in characterizing a European collection of epidemic methicillinresistant Staphylococcus aureus strains: the HARMONY collection. J Clin Microbiol 2007;45:1830-1837.

20 Smyth AR, Bell SC, Bojcin S, Bryon M, Duff A, et al: European Cystic Fibrosis Society Standards of Care: Best Practice guidelines. J Cyst Fibros 2014;13:23-42.

21 Luedicke C, Slickers P, Ehricht R, Monecke S: Molecular fingerprinting of Staphylococcus aureus from bone and joint infections. Eur J Clin Microbiol Infect Dis 2010;29:457-463.

22 Monecke S, Luedicke C, Slickers P, Ehricht R: Molecular epidemiology of Staphylococcus aureus in asymptomatic carriers. Eur J Clin Microbiol Infect Dis 2009;28:1159-1165.

23 Rolo J, Miragaia M, Turlej-Rogacka A, Empel $\mathrm{J}$, Bouchami O, et al: High genetic diversity among community-associated Staphylococcus aureus in Europe: results from a multicenter study. PLoS One 2012;7:e34768.

24 Hirschhausen N, Block D, Bianconi I, Bragonzi A, Birtel J, et al: Extended Staphylococcus aureus persistence in cystic fibrosis is associated with bacterial adaptation. Int J Med Microbiol 2013;303:685-692. 